\title{
Correlação entre o tempo para a primeira sedestação beira leito e o tempo de internação hospitalar em vítimas de traumatismo cranioencefálico
}

\author{
Correlation between bedside sedestation time and hospitalization time for victims of \\ cranioencephalic traumatism
}

Corelación entre el tiempo de sedestación de cabecera y tiempo de hospitalización hospitalaria em víctimas de traumatismo craniencefálico internados

Jamylle Silva Campos ${ }^{1 *}$, Ellen do Socorro Cruz de Maria $^{2}$, Aline Silva Castro², Diane Elen Damasceno Queiroz ${ }^{1}$, Blenda Danielly Libdy Miranda ${ }^{1}$, Rafael Ângelo Araújo ${ }^{3}$, Rodrigo Santiago Barbosa Rocha ${ }^{4}$, Lucas Monteiro Carneiro ${ }^{5}$, Daniel da Costa Torres ${ }^{6}$.

\section{RESUMO}

Objetivo: Verificar a influência do tempo que a primeira sedestação de pacientes vítimas de Traumatismo Cranioencefálico (TCE) tem sobre o tempo total de internação na Unidade de Terapia Intensiva (UTI) e hospitalar. Métodos: Foram analisados prontuários de pacientes com idade entre 18 a 70 anos, de ambos os sexos, vítimas de TCE, que deram entrada no hospital, com necessidade de intubação e internação na UTI, no período de dezembro de 2019 à novembro de 2020. Foram coletados a data de internação e alta hospitalar, da primeira sedestação beira leito, ortostatismo e marcha. Foi feita uma análise estatística descrita, seguida da aplicação do teste do Qui-quadrado e para correlação utilizou o Teste de Correlação Linear de Pearson. Resultados: 132 prontuários atenderam os critérios de inclusão. $85,60 \%$ eram do sexo masculino com idade média de 35,05 $\pm 12,93$ anos, 51,52\% tiveram como mecanismo de lesão acidente de moto e $65,15 \%$ foram classificados como TCE grave. Obteve-se um coeficiente de correlação fraco - 0,1705 e - $-0,1195$, com um pvalor $>0,05$ em relação ao tempo da primeira sedestação beira leito com tempo de internação da UTI e hospitalar. Conclusão: Nota-se que não há influência do tempo levado para a primeira sedestação com o tempo de internação na UTI e hospitalar total.

Palavras-chave: Fisioterapia, Hospitalização, Lesões encefálicas traumática.

\begin{abstract}
Objective: To verify the correlation of time for the first sedestation of a patient suffering from Cranioencephalic Traumatism (TBI) has an influence on the length of stay in the Intensive Care Units (ICU) and hospital. Methods: Medical records of patients aged 18 to 70 years, of both genders, victims of TBI, who were admitted to the hospital, requiring intubation and ICU admission, from December 2019 to November 2020, were included. The date of admission and hospital discharge, first bedside sedation, orthostatism and gait were collected. A descriptive statistical analysis was perfomed, followed by the Chi-square test application and, for correlation, was used the Pearson1s Linear Correlation Test. Results: 132 medical records met the inclusion
\end{abstract}

\footnotetext{
${ }^{1}$ Centro Universitário do Estado do Pará, Belém - PA. *E-mail: jamylle.campos@hotmail.com

2 Universidade do Estado do Pará, Belém - PA.

${ }^{3}$ Fundação Hospital de Clínicas Gaspar Vianna, Belém - PA.

${ }_{4}$ Universidade Metodista de Piracicaba, Piracicaba - SP.

${ }^{5}$ Hospital Metropolitano de Urgência e Emergência, Belém - PA.

${ }^{6}$ Universidade Cidade de São Paulo, São Paulo - SP.
}

SUBMETIDO EM: 2/2021

PUBLICADO EM: 4/2021 
criteria. $85.60 \%$ were male with an average age of $35.05 \pm 12.93$ years, $51.52 \%$ had a motorcycle accident as an injury mechanism and $65.15 \%$ were classified as severe TBI. A weak correlation coefficient of -0.1705 and -0.1195 was obtained, with a $p$-value $>0.05$ in relation to the time of the first bedside sedestation with length of stay in the ICU and hospital. Conclusion: It is noted that there is no correlation between the time of the first sedestation and the length of stay in the ICU and the total hospital stay.

Key words: Physical therapy specialty, Hospitalization, Brain injuries traumatic.

\section{RESUMEN}

Objetivo: Verificar la correlación del tiempo para la primera sedestación de paciente víctima de Traumatismo Craniencefálico (TCE) tiene influencia sobre el tiempo de internación en la Unidades de Cuidados Intensivos (UCl) y hospitalaria. Métodos: Fueran incluidos prontuarios de pacientes con edad de 18 a 70 años, de ambos los sexos, víctimas de TC, que dieran entrada en el hospital, con necesidad de intubación e internación en la $\mathrm{UCl}$ a lo largo de los meses de diciembre de 2019 a noviembre de 2020. Se recogieron fecha de ingreso y alta hospitalaria, primera sedación de cabecera, ortostatismo y marcha. Fue realizado un análisis estadístico descriptivo, seguido de la aplicación de la prueba de Chi-cuadrado y, para la correlación, se utilizó la Prueba de Correlación Lineal de Pearson. Resultados: 132 prontuarios atendieron los criterios de inclusión. Siendo que, $85,60 \%$ eran del sexo masculino con una media de edad $35,05 \pm 12,93$ años, $51,52 \%$ tuvieron como mecanismo de lesión el accidente de motocicleta e $65,15 \%$ fueron clasificados como TCE grave. Se obtuvo un coeficiente de correlación débil - 0,1705 e - -0,1195, con un $p$ - valor>0,05 en relación con el tiempo de la primera sedestación de cabecera con tiempo de internación de la UCl y hospitalaria. Conclusión: Se observa que no hay influencia entre el tiempo de la primera sedestación con el tiempo en la UCl y hospitalaria total.

Palabras clave: Fisioterapia, Hospitalización, Lesiones traumáticas del encéfalo.

\section{INTRODUÇÃO}

O Traumatismo Cranioencefálico (TCE) é a principal causa de morte e incapacidade física no mundo, gerando altos custos para a saúde pública além de consideráveis prejuízos à qualidade de vida dos indivíduos acometidos. Estes traumas podem causar danos cerebrais temporários ou permanentes, classificados como leve, moderado ou grave. Os casos graves frequentemente demandam cuidados contínuos e intensivos, necessitando de internação em Unidade de Terapia Intensiva (UTI) e uso da Ventilação Mecânica Invasiva (VMI) (PADOVANI C, 2015; JOHNSON WS e GRISWOLD DP, 2017).

Para estes pacientes, a VMI é indicada visando a proteção das vias aéreas e controle das pressões parciais de oxigênio e gás carbônico ( $\mathrm{PaO} 2$ e $\mathrm{PO} 2$ ) buscando prevenir injúrias cerebrais secundárias ao trauma. Apesar de não possuírem lesões pulmonares, frequentemente, no momento da internação na UTI, evidencia-se que estes indivíduos evoluem com tempo prolongado de ventilação mecânica e desmame difícil, se comparados a outras populações em cuidados intensivos. Tais adversidades levam a disfunções respiratórias e musculoesqueléticas, destacando-se o dano muscular decorrente de inatividade física, processos inflamatórios e uso de fármacos (CINOTTI R, et al., 2018; COUTINHO W, et al., 2016; REIS HFC, et al., 2013).

Ademais, durante esse período de internação o indivíduo fica sujeito às complicações resultantes do imobilismo no leito. Tais distúrbios estão relacionados a alterações de natureza física e psicossociais, como úlceras de pressão, isolamento e humor deprimido, além de diversos efeitos provocados nos demais sistemas como: cardiovascular, respiratório, gastrointestinal e urinário. A soma destes fatores ocasiona um impacto importante na funcionalidade destes pacientes, responsáveis por $30 \%$ dos casos de incapacidades transitórias após a internação, além de aumentar o risco de incapacidade permanente (FORMENTI P, et al., 2019; GUEDES L, et al., 2018; SARTI TC, et al., 2016).

Nesse sentido, a realização da sedestação beira leito, bipedestação e deambulação desse paciente durante a internação na UTI, é uma das formas de mobilização precoce. Sendo uma terapêutica eficaz e 
fundamental para o paciente critico, visto que promove benefícios físicos e psicológicos, evita complicações decorrentes do tempo de hospitalização prolongado e síndrome do imobilismo, apresentando como principais respostas a diminuição no tempo de internação e redução da incidência de complicações pulmonares e funcionais. Portanto, pesquisas que visem o estudo dos benefícios e aplicação desta técnica, são de grande valia para a fisioterapia em pacientes vítimas de TCE (AQUIM EE, et al., 2019; SILVA PF, et al., 2014; SARTI TC, et al., 2016).

Sendo assim, o objetivo do estudo foi correlacionar a influência do tempo para a realização da primeira sedestação de paciente vítima de TCE tem sobre o tempo de internação na UTI e hospitalar.

\section{MÉTODOS}

Trata-se de uma pesquisa de caráter observacional, transversal, retrospectivo e quantitativo. Aprovado pela Comité de Ética e Pesquisa do Centro Universitário do Estado do Pará, sob parecer 4.371.360.

A coleta de dados foi realizada em um Hospital referência na região Norte, em atendimentos de média e alta complexidade em traumas, localizado no estado do Pará. Onde foi realizado uma busca ativa em prontuários eletrônicos de pacientes vítimas de TCE, com idade entre18 a 70 anos, de ambos os sexos, que deram entrada do hospital, com necessidade de intubação e internação na UTI, durante os meses de dezembro de 2019 à novembro de 2020. Adotando como critérios de exclusão prontuários de pacientes que foram a óbito, que tiveram $20 \%$ de preenchimento incompleto, que possuíam outro diagnóstico clínico associado ao TCE e sem necessidade de VM.

A busca ativa foi realizada nos indicadores eletrônicos do Departamento de Reabilitação do Hospital, após a liberação e a disponibilização dos dados pelo Coordenador do setor, com enfoque apenas aos dados referentes as duas UTI do serviço, as quais possuíam 10 leitos cada. Foram coletadas informações acerca da idade, sexo, mecanismo do trauma, data de admissão hospitalar, Escala de Coma de Glasgow (ECG) de admissão, forma de tratamento (cirúrgico ou conversador), data de admissão na UTI, tempo de intubação orotraqueal, traqueostomia, tempo para decanulação, tempo de ventilação mecânica, data e tempo para realização da primeira sedestação, ortostatismo e marcha, além disso, data de alta da UTI e alta hospitalar. Todos os dados coletados foram tabulados em ficha própria, sendo a coleta realizada durante o período da tarde, com uma frequência de 3 vezes semanais.

Para a análise estatística foi utilizado o Software BioEstat ${ }^{\circledR} 5.0$ (Sociedade Civil Mamirauá, Manaus, Brasil). Inicialmente, foi realizado o teste de normalidade D'Agostino-Pearson, seguido de uma análise estatística descritiva, para estudo da distribuição das variáveis.

Posteriormente, para as variáveis nominais, houve distribuição em tabelas em forma de frequência simples e relativas (porcentagens), seguida da aplicação do teste do Qui-Quadrado. As variáveis numéricas foram apresentadas por meio de medidas de tendência central (média) e dispersão (desvio padrão) e as demais através de mediana e de primeiro e terceiro intervalo interquartílico (IQ).

A fim de correlacionar o tempo da primeira sedestação com o tempo de internação hospital e de UTI foi adotado o Teste de Correlação Linear de Pearson, considerando-se um alfa de significância de $5 \%(p \leq 0,05)$.

\section{RESULTADOS}

Foram analisados 176 prontuários, dos quais 39 foram excluídos por óbito durante o período analisado e 5 por não adequação aos critérios de inclusão, totalizando uma amostra de 132 pacientes. Desses, a predominância foi do sexo masculino (85,60\%), com uma média de idade $35,05 \pm 12,93$ anos, que tiveram como mecanismo de lesão acidente de moto $(51,52 \%)$ e advindos principalmente da Região Metropolitana de Belém (54.55\%). Em relação a pontuação na Escala de Coma de Glasgow Inicial, 65,15\% dos pacientes obtiveram a pontuação entre 3 a 8 , classificados como TCE grave, sendo submetidos ao tratamento conservador em $63,64 \%$ dos casos (Tabela 1 ). 
Tabela 1 - Características dos pacientes atendidos na Unidade de Terapia Intensiva de um Hospital de Urgência e Emergência.

\begin{tabular}{|c|c|c|c|}
\hline \multirow{2}{*}{ Variáveis } & \multicolumn{3}{|c|}{ Participantes ( $\mathrm{N}=132$ ) } \\
\hline & $\mathbf{N}$ & $\%$ & p-Valor \\
\hline \multicolumn{4}{|l|}{ Sexo } \\
\hline Masculino & 113 & 85,60 & \multirow{2}{*}{$0,001^{*}$} \\
\hline Feminino & 19 & 14,40 & \\
\hline \multicolumn{4}{|l|}{ Local } \\
\hline Região Metropolitana & 72 & 54,55 & \multirow{2}{*}{0,61} \\
\hline Interior & 60 & 45,45 & \\
\hline \multicolumn{4}{|l|}{ Mecanismo de Lesão } \\
\hline Acidente de Carro & 6 & 4,54 & \multirow{8}{*}{ - } \\
\hline Acidente de Moto & 68 & 51,52 & \\
\hline Agressão Física & 14 & 10,60 & \\
\hline Atropelamento & 17 & 12,87 & \\
\hline Ferimento por Arma de Fogo & 7 & 5,30 & \\
\hline Queda de Altura & 5 & 3,80 & \\
\hline Queda da Própria Altura & 5 & 3,80 & \\
\hline Outros & 10 & 7,57 & \\
\hline \multicolumn{4}{|l|}{ Tratamento } \\
\hline Conservador & 84 & 63,64 & \multirow{2}{*}{$0,026^{*}$} \\
\hline Cirúrgico & 48 & 36,36 & \\
\hline \multicolumn{4}{|l|}{ Escala de Coma de Glasgow (ECG) } \\
\hline 3 a 8 & 86 & 65,15 & \multirow{3}{*}{$0,039^{*}$} \\
\hline 9 a 13 & 27 & 20,45 & \\
\hline 14 a 15 & 19 & 14,40 & \\
\hline \multirow{2}{*}{ Idade (anos) } & Média & DP & \multirow{2}{*}{-} \\
\hline & 35,05 & $\pm 12,93$ & \\
\hline
\end{tabular}

Legenda: Frequências simples e relativas (porcentagens) dos dados, com média e desvio padrão (DP) de variáveis paramétricas. P-valor obtido pelo teste de Qui-Quadrado, $\left({ }^{*}\right)$ mostra o valor de $p<0,05$, como significante.

Fonte: Campos JS, et al, 2021.

Em relação a frequência de procedimentos realizados, nota-se que $72(54,55 \%)$ não necessitaram de reintubação em $48 \mathrm{~h}$ após retirada do tubo orotraqueal (TOT), e $58(43,93 \%)$ passaram pelo procedimento cirúrgico de traqueostomia.

Entretanto, 51 (87,94\%) desses participantes realizaram a decanulação ainda durante o período de internação hospitalar. No que diz respeito aos aspectos de mobilização precoce dentro da UTI, $126(87,94 \%)$ realizaram a sedestação beira leito, porém 78 (59,10\%) não realizaram bipedestação e 104 (78,78\%) não realizaram deambulação (Tabela 2). 
Tabela 2 - Frequência dos pacientes em relação aos procedimentos realizados durante o período de Internação Hospitalar e na UTI.

\begin{tabular}{|c|c|c|c|}
\hline \multirow{2}{*}{ Variáveis } & \multicolumn{3}{|c|}{ Participantes ( $\mathrm{N}=132)$} \\
\hline & $\mathbf{N}$ & $\%$ & p-Valor \\
\hline \multicolumn{4}{|c|}{ Sucesso de Extubação? \# } \\
\hline Sim & 72 & 54,55 & \multirow{2}{*}{$0,001^{*}$} \\
\hline Não & 2 & 1,52 & \\
\hline Traqueostomizados" ${ }^{\#}$ & 58 & 43,93 & \\
\hline \multicolumn{4}{|l|}{ Decanulação" } \\
\hline Sim & 51 & 87,94 & \multirow{2}{*}{$0,001^{*}$} \\
\hline Não & 7 & 12,06 & \\
\hline \multicolumn{4}{|c|}{ Realizou Sedestação Beira Leito?\# } \\
\hline Sim & 126 & 95,46 & \multirow{2}{*}{$0,001^{*}$} \\
\hline Não & 6 & 4,54 & \\
\hline \multicolumn{4}{|l|}{ Realizou Ortostase?" } \\
\hline Sim & 54 & 40,90 & \multirow{2}{*}{$0,035^{\star}$} \\
\hline Não & 78 & 59,10 & \\
\hline \multicolumn{4}{|l|}{ Fez Treino de Marcha?\# } \\
\hline Sim & 28 & 21,22 & \multirow{2}{*}{$0,001^{*}$} \\
\hline Não & 104 & 78,78 & \\
\hline
\end{tabular}

Legenda: Frequências simples e relativas (porcentagens) dos dados, com média e desvio padrão (DP) de variáveis paramétricas. P-valor obtido pelo teste de Qui-Quadrado, $\left({ }^{*}\right)$ mostra o valor de $p<0,05$, como significante. (\#) mostra os procedimentos realizados no período de internação na UTI. (") representa procedimento realizado enfermaria. Fonte: Campos JS, et al, 2021.

Ao analisar-se o tempo de TOT, notou-se a média de 7,71 $\pm 3,48$ dias, inferior ao tempo de VM que foi de 8 (5-12) dias. Quanto ao tempo para decanulaçao, apresentou-se a mediana de 19 (12-25,5). Já em relação ao tempo para realização da primeira sedestação beira leito, bipedestação e deambulação, teve como mediana, respectivamente, 6 (4-9), 7 (5-11,75) e 7,5 (5-11). O tempo total de internação na UTI foi de 11 (714,50) dias e o tempo de internação hospitalar foi de 25 (17-37) dias (Tabela 3).

Tabela 3 - Tempo (em dias) de internação na UTI e hospitalar e o tempo de procedimentos e mobilização dos pacientes vítimas de TCE.

\begin{tabular}{|c|c|c|}
\hline \multirow{2}{*}{ Variáveis } & \multicolumn{2}{|c|}{ Participantes ( $\mathrm{N}=132)$} \\
\hline & Média & DP \\
\hline Tempo de Tubo Orotraqueal (dias) & 7,71 & $\pm 3,48$ \\
\hline Tempo para Realização da TQT (dias) & 9,72 & $\pm 2,72$ \\
\hline & Mediana & $I Q\left(1^{\circ}-3^{\circ}\right)$ \\
\hline Tempo de Ventilação Mecânica (dias) & 8,00 & $5-12$ \\
\hline Tempo de Desmame de TQT (dias) & 19 & $12-25,5$ \\
\hline 1은 Sedestação Beira Leito (dias) & 6 & $4-9$ \\
\hline $1^{\circ}$ Ortostase (dias) & 7 & $5-11,75$ \\
\hline 10 Treino de Marcha (dias) & 7,5 & $5-11$ \\
\hline Tempo Total UTI (dias) & 11 & $7-14,50$ \\
\hline Tempo Internação Hospitalar (dias) & 25 & $17-37$ \\
\hline $\begin{array}{l}\text { Legenda: TQT - Traqueostomia. U } \\
\text { Intensiva. DP - Desvio Padrão. IQ - Int } \\
\text { Campos JS, et al, } 2021 .\end{array}$ & & $\begin{array}{l}\text { le Terapia } \\
\text { ico. Fonte: }\end{array}$ \\
\hline
\end{tabular}


Em referência a correlação entre o tempo levado para realizar a primeira sedestação beira leito e o tempo de internação na UTI e o tempo de internação Hospitalar Total, nota-se um coeficiente de correlação fraco 0,1705 e - -, 1195 , com um p-valor >0,05, logo o tempo para a realização do primeira sedestação não interfere no tempo de internação do paciente TCE, tanto a nível de UTI quanto a hospitalar (Tabela 4).

Tabela 4 - Correlações entre o Tempo para a primeira sedestação e os tempos de internação na UTI e hospitalar.

\begin{tabular}{cccc}
\hline Variáveis & $\begin{array}{c}\mathbf{r} \\
\text { (Pearson) }\end{array}$ & IC 95\% & p- valor \\
\hline Sedestação x Tempo de UTI (dias) & $-0,1705$ & $-0,36$ a 0,03 & 0,098 \\
\hline $\begin{array}{c}\text { Sedestação x Tempo de Internação } \\
\text { Hospitalar (dias) }\end{array}$ & $-0,1195$ & $-0,31$ a 0,08 & 0,248 \\
\hline
\end{tabular}

Legenda: IC - Intervalo de Confiança. Fonte: Campos JS, et al, 2021.

\section{DISCUSSÃO}

Os achados da pesquisa corroboram com os dados presentes na literatura atual, a qual aponta um predomínio do sexo masculino entre as vítimas de TCE, com idade, em média 35,05 $\pm 12,93$, e local dos acidentes corridos, 54,55\% na região metropolitana. Para Marinho et al. 2019 está predominância se dá devido ao comportamento característico deste grupo, o qual tende a se expor de maneira mais frequente a riscos devido a atração pela velocidade, motivados pelo contexto social e estilo de vida que ocasionalmente fazem a combinação de álcool e direção (SILVA TH, et al., 2018).

Os acidentes de trânsito são uma das principais causas de morbimortalidade na população, sendo os motociclistas os públicos mais vulneráveis e propensos a estas ocorrências. Tal fato pode estar relacionado com o aumento do número da frota de motocicletas no país, visto que, elas apresentam um menor custo e maior agilidade quando comparada a outros meios de transporte, principalmente nas regiões metropolitanas. Além disso, este é um meio de locomoção frequentemente escolhido pelos jovens, que por vezes apresentam comportamento de direção de perigosa e imprudente, contribuindo para um maior número de acidentes (BONOW RH, et al., 2018; BITTAR CK, et al., 2020).

A respeito da classificação da gravidade do TCE, verificada mediante a constatação do nível de consciência pela ECG, onde $65,15 \%$ obteve o score variando de 3 a 8 , sendo classificado como TCE grave. Assim, necessitando de intubação orotraqueal, afim de controlar a Pressão Intracraniana (PIC), evitar hipoxemia, herniações e lesões secundárias, além de proteção das Vias Aéreas Superiores (VAS) (AIMB, 2013).

O trauma gera um edema cerebral, no qual acarreta um aumento da PIC e redução da pressão de perfusão cerebral (PPC), com isso afetando o metabolismo cerebral e consumo de oxigênio. Desse modo, duas medidas terapêutica podem ser realizadas, sendo a primeira abordagem o tratamento conservador, que envolve otimização da sedação, monitorização da $\mathrm{PIC}<20 \mathrm{mmHg}$, VM que proporcione uma Pressão Parcial de Dióxido de Carbono (PaCO2) entre 30 a $35 \mathrm{mmHg}$, verificação da pressão venosa central, osmoterapia, posicionamento da cabeceira a 30 graus com a cabeça em posição neutra, para melhora do retorno venoso e acompanhamento pelo Tomografia Computadorizada (AIMB, 2013; FÁTIMA N, et al., 2019; PADOVANI C, et al., 2017).

Tal protocolo é adotado pelo Hospital do estudo, sendo associado a avaliação do diâmetro da pupila a cada 2 horas, onde toda a equipe multiprofissional é responsável pela verificação. Cada profissional é elencado para um horário pré-definido durante seu plantão, após avaliação, anota sua avaliação em uma ficha individualizada do paciente.

Quando tais medidas não conseguem controlar a PIC, opta-se pelo procedimento cirúrgico, denominado de craniectomia descompressiva, que permite um alívio instantâneo e permanente da pressão. Porém, ainda não há consenso sobre qual período para executar a cirurgia, para que ocasione um melhor resultado clínico do paciente (FÁTIMA N, et al., 2019; SOUSA CDD, et al., 2020). 
No estudo de Silva TH, et al. (2018), mostra as repercussões da gravidade do TCE verificado na admissão hospitalar sobre os resultados clínicos, onde teve uma amostra 67 indivíduos, constatou que 53,7\% teve TCE leve e apenas 25,4\% TCE grave, divergindo do estudo atual. Mas em relação ao tratamento adotado frente a estes pacientes, nota-se dados semelhante, pois $76,1 \%$ foram submetidos ao tratamento conservador e $23,9 \%$ ao tratamento cirúrgico. Sendo que, neste estudo, a abordagem do tratamento conservador ocorreu $63,64 \%$ e apenas $36,36 \%$ realizaram neurocirúrgia.

Nota-se que a gravidade do TCE faz com que o paciente demande mais atenção e procedimentos, assim sofrendo repercussões tanto devido ao trauma do Sistema Nervoso Central (SNC), assim como devido o tempo de intubação, VM, cirurgias, presença de sondas, imobilidade no leito, lesões por pressão, entre outros. Gerando grandes repercussões na reabilitação desse paciente pós alta hospitalar.

Em relação ao processo de extubação, houve sucesso em $54,55 \%$ da amostra, assim pode se perceber que após 48 horas, os pacientes permaneceram em respiração espontaneamente sem necessidade de suporte ventilatório. Contudo, $43,93 \%$ necessitaram de realizar procedimento cirúrgico de traqueostomia. A extubação é um processo que necessita da estabilização do quadro clínico, nível de consciência do paciente, drive respiratório satisfatório, sucesso no Teste de Respiração Espontânea (TRE), proteção das vias aéreas, entre outros fatores, para garantir seu sucesso. Enquanto que, a falha desse processo associa-se com desfechos negativos, como aumento do tempo de permanência na UTI e internação total, além de elevar os níveis de mortalidade e morbidade, número de traqueostomizados e custo hospitalar (AMIB, 2013; MEDEIROS ACS, et al., 2015; SILVA VS, et al., 2018).

Nesses sentindo, o tempo de TOT, assim como o tempo de permanência na UTI desse estudo corrobora com os dados de Nascimento S, et al. (2020), que descrever a epidemiologia do paciente TCE grave de pacientes admitidos em uma UTI. Nota-se também que o procedimento de TQT teve uma incidência de $43 \%$ em paciente TCE, tal fato ocorre devido a incapacidade que a lesão ocasiona, como déficit na deglutição, fala, disfagia, diminuição do tônus muscular, entre outros, assim gerando obstrução das VAS, aumentando os riscos de broncoaspiração e tornando o desmame difícil (AMIB, 2013; LIM CK, et al., 2015; MOTA JDH, et al., 2020).

Observou-se nesse estudo a incidência de TCE grave, com tempo de realização da TQT em média de $9,72 \pm 2,72$ dias, aproximadamente o tempo indicado na literatura para o procedimento, sendo de 7 dias de TOT, com perspectiva de VM prolongada. Assim, observa-se que a traqueostomia precoce em pacientes neurológicos, tem como finalidade permitir uma melhor proteção da VAS, menor taxa de pneumonia associada a VM (PAV), diminuição do tempo e dependência da VM, como menor tempo de internação na UTI (AMIB, 2013; MOTA JDH, et al, 2020).

Pode ser ressaltar que o processo de decanulação da TQT também interfere no tempo de hospitalização total, ocorrendo período de internação na enfermaria, sendo realizado de forma multiprofissional no HMUE, com participação do fisioterapeuta, fonoaudiólogo, médico e enfermeiro, proporcionando aceleração da retirada da prótese, melhora da qualidade de vida, fonação, deglutição e tosse (BOULHOSA FJS, et al., 2015). Zanata IL, et al. (2016), verificou que o grupo submetido ao protocolo terapêutico com fonoaudiológo teve um menor tempo de permanência de TQT $(19,2 \pm 10,3)$ e menor tempo de internação hospitalar $(28,7 \pm 10,8)$, quando comparado ao grupo controle $(23,2 \pm 12,4$ e $33,1 \pm 15,3$, respectivamente) corroborando com os achados desse estudo.

A longa estadia hospitalar do paciente TCE provoca diversas complicações, ao nível musculoesquelético e funcional, observa-se que em 7 dias de repouso ocorre uma perda aproximadamente de $30 \%$ da força muscular, sendo tal perda progressiva, quando o estado de imobilidade permanece. Tal fato, acontece constantemente com o paciente neurocrítico e sob VM, ocasionando aumento do tempo de intubação, ventilação prolongada, maior taxa de traqueostomia, tempo de internação, custos hospitalares e diminuição da funcionalidade e qualidade de vida (SIBINELLI M, et al., 2012; THOMAS e MEHRHOLZ J, 2018).

Assim, a Diretriz Brasileira de Ventilação Mecânica (2013) relata que a mobilização precoce deve ser realizada com o paciente sobre VM, assim como após o processo de extubação do mesmo. Sendo considera 
um procedimento seguro e associado a melhoras da funcionalidade do indivíduo, como menor tempo sobre VM e internação. Onde ocorre mediante atividades sensório motoras, posicionamento adequado, eletroestimulação, alongamentos, exercício passivo, ativo, ativo-assistido, resistivo e atividades funcionais, como sedestação beira leito, bipedestação e treino de transferência e marcha, entre outras modalidades (PADOVANI C, et al., 2017; SILVA PE, et al., 2019).

Com referência a sedestação beira leito dos pacientes vítimas de TCE, 95,46\% ( $p=0,001)$ realizaram tal procedimento, porém $59,10 \%(p=0,035)$ e $78,78 \%(p=0,001)$ dos pacientes, respectivamente, não realizaram ortostatismo e deambulação durante o período de internação na UTI. Tal fato, pode ocorrer devido as incapacidades funcionais, déficits de força, alteração do tônus muscular e das atividades cognitivas. Assim como, devido ao desmame prolongado (THOMAS S e MEHRHOLZ J, 2018; SOUSA CDD, et al., 2020).

As barreiras presentes dentro da UTI interferem na realização da sedestação, ortostase e deambulação do paciente TCE, podendo elencar como, barreiras clínicas: baixo nível de consciência, falta de colaboração, presença de dor, alterações hemodinâmicas e metabólicas. Barreiras para o fisioterapeuta: alta demanda de trabalho, intercorrências, fatores ergonômicos, falta de apoio da equipe de trabalho. Por fim, barreira da unidade: falta de equipamentos que auxiliem e facilitem nessa mobilização, como prancha ortostática.

No que diz respeito a análise da interferência do tempo da primeira sedestação sobre o tempo de internação na UTI e hospitalar, obteve-se uma correlação fraca entre ambas as variáveis. Feliciano VA, et al. (2012), constatou também, que a mobilização precoce em pacientes críticos não interferiu no tempo de VM, UTI ou hospitalar total, porém houve melhora nas variáveis força muscular inspiratória e periférica, além de melhora da funcionalidade no momento da alta da UTI.

Enquanto, Pang $\mathrm{Y}$, et al. (2019), avaliou a eficácia e segurança da terapia de reabilitação precoce abrangente para pacientes de UTI, com diagnóstico de hemorragia cerebral ou lesão cerebral traumática, possuindo amostra de 42 indivíduos. O grupo submetido a terapia de reabilitação precoce aumentou a força muscular global, melhorou o nível de consciência dos pacientes da UTI, diminuiu a incidência de complicações e encurtou a permanência total no hospital e o tempo de ventilação mecânica dos pacientes na UTI.

Analisando a introdução de um fisioterapeuta na equipe de terapia intensiva, específico para reabilitação do paciente crítico. Traçando uma estratégia de reabilitação precoce centrada no paciente ventilados mecanicamente por mais de 5 dias, ocasionou redução nos dias de VM, tempo de UTI e total de internação hospitalar. Além de, melhora a função física no momento de alta da UTI. Assim, considera que, a mobilização possibilitou uma melhor e/ou manutenção da funcionalidade (MWILLIAM, 2015; MURAKAMI FM, et al., 2015).

Ainda não há um consenso sobre a interferência da mobilização precoce sobre o tempo de internação do paciente TCE, porém observa-se que esta possui um efeito positivo na funcionalidade, capacidade respiratória e musculoesquelética. Ocasionando menores repercussões da internação na qualidade de vida e atividades de vida diária após a saída do ambiente hospitalar.

Elenca-se como limitação do estudo, ausência de dados sobre o aspecto funcional desses pacientes durante sua internação, para mensurar a qualidade e os efeitos dos procedimentos realizados. Também vale ressaltar que o estudo ocorreu no período de pandemia do COVID-19, que pode ter afetado o número total de paciente vítimas de TCE no período estudado, devido ao período de diminuição da circulação de pessoas, gerando ou não um menor número de acidentes automobilístico, já que foi observado como a principal mecanismo de trauma. Além disso, não foram coletados dados referentes a suspeita ou confirmação de diagnóstico para coronavírus, que pode ter interferido ao tempo de internação e tubo orotraqueal desses pacientes.

\section{CONCLUSÃO}

Constata-se não há influência do tempo para a realização da primeira sedestação sobre o tempo de internação na UTI e hospitalar. Tal resultado pode ser devido a necessidade outros dados para subsidiar o estudo, como: grau de funcionalidade pré e pós mobilização e frequência e repetição dos atendimentos fisioterapêuticos. Assim, necessitando de novos estudos, afim de determinar fatores que proporcione um 
menor tempo de hospitalização. Porém, os fatores identificados permitem traçar um perfil clínico do paciente vítima de TCE atendidos em um hospital de referência em trauma do Estado do Pará.

\section{AGRADECIMENTOS}

Agradecemos ao Setor de Fisioterapia do Hospital que ajudou e facilitou a coleta de dados, assim como aos funcionários do Departamento de Ensino e Pesquisa, Natacha Mariana Farias da Cunha e Andrey Abdon de Sousa, que acolheram os pesquisadores e apoiaram o processo de pesquisa.

\section{REFERÊNCIAS}

1. AQUIN EE, et al. Diretrizes Brasileiras de Mobilização Precoce em Unidade de Terapia Intensiva. Ver bras ter intensiva, 2020; 31(4): 434-443.

2. BITTAR CK, et al., 2020. Epidemiological Profile of Motorcycle Accident Victims in University Hospital. Acta Ortop Bras, 2020;28(2):97-9.

3. BOULHOSA FJS, et al. O Impacto do protocolo de desmame de traqueostomia em pacientes vítimas de Traumatismo Cranioencefálico internados no Hospital Metropolitano de Urgência e Emergência no Pará. Rev Univ Val R Verde, 2015; (13)2: 313-323.

4. CINOTTI R, et al. Management and weaning from mechanical ventilation in neurologic patients. Ann TransI Med. 2018; 6(19):381.

5. COUTINHO W, et al. Efeito agudo da utilização do cicloergômetro durante atendimento fisioterapêutico em pacientes críticos ventilados mecanicamente. Fisioter Pesqui, 2016;23(3):278-283.

6. FÁTIMA N, et al. The Role of Decompressive Craniectomy in Traumatic Brain Injury: A Systematic Review and Metaanalysis. Asian J Neurosurg, 2019; 14(2): 371-381.

7. FELICIANO VA, et al. A influência da mobilização precoce no tempo de internamento na Unidade de Terapia Intensiva. ASSOBRAFIR Ciência, 2012;3(2):31-42.

8. FORMENTI P, et al. Clinical review: peripheral muscular ultrasound in the ICU. Ann Intensive Care, 2019; 57(9).

9. GUEDES L, et al. Efeitos deletérios do tempo prolongado no leito nos sistemas corporais dos idosos - uma revisão. Rev bras geriatr gerontol, 2018; 21(4):499-506.

10. HASSETT L, et al. Fitness training for cardiorespiratory conditioning aer traumatic brain injury (Review). Cochrane Database Syst Rev, 2017; 12 (12).

11. JOHNSON WS, GRISWOLD DP. Traumatic brain injury: a global challenge. Lancet Neurol. 2017;16(12):949-950.

12. LIM CK, et al. Effect of tracheostomy on weaning parameters in difficultto- wean mechanically ventilated patients: a prospective observational study. PLoS ONE, 2015;10(9).

13. MARINHO CRS, et al. Acidente de trânsito: análise dos casos de traumatismo cranioencefálico. Enfermaria Global, 2019; 54: 333-342.

14. MEDEIROS ACS, et al. Perfil clínico e índices preditivos de desmame de pacientes extubados em uma unidade de terapia intensiva de Fortaleza, CE. ASSOBRAFIR Ciência, 2015; 6(3):33-42.

15. MOTA JDH, et al. Análise do tempo de retirada do respirador artificial no paciente submetido a traqueostomia precoce e após sete dias de ventilação mecânica invasiva. Fisioter Pesqui. 2020;27(3):306-31.

16. MURAKAMI FM, et al. Evolução funcional de pacientes graves submetidos a um protocolo de reabilitação precoce. Rev Bras Ter Intensiva, 2015; 27(2):161-169.

17. NASCIMENTO S, et al. Perfil epidemiológico de pacientes adultos com traumatismo cranioencefálico grave na rede SUS do Distrito Federal: um estudo retrospectivo. Rev Bras Neurol, 2020; 56(4):5-10.

18. PADOVANI C, et al. Physiotherapy in severe polytrauma patients: a therapeutic care model. Acta Fisiatr, 2017;24(1):33-39.

19. PADOVANI, C. Fisioterapia Respiratória no Traumatismo Cranioencefálico: Revisão de Literatura. Rev Insp Mov Saud, 2015; 7(3).

20. PANG Y, et al. An Established Early Rehabilitation Therapy Demonstrating Higher Efficacy and Safety for Care of Intensive Care Unit Patients. Med Sci Monit, 2019; 25: 7052-7058.

21. REIS HFC, et al. Associação entre o índice de respiração rápida e superficial e o sucesso da extubação em pacientes com traumatismo cranioencefálico. Rev bras ter intensiva, 2013; 25(3):212-217.

22. SARTI TC, et al. Mobilização precoce em pacientes críticos. J Health Sci Inst, 2016; 34(3):177-82.

23. SIBINELLI M, et al. The effects of orthostatism in adult intensive care unit patients. Rev bras ter intensiva [online], 2012; 24(1): 64-70.

24. SILVA PF, et al. Caracterização das vítimas de traumatismo encefálico que evoluíram para morte encefálica. Rev Cuid, 2018; 9(3): 2349-60.

25. SILVA TH, et al. Influence of severity of traumatic brain injury at hospital admission on clinical outcomes. Fisioter. Pesqui, 2018; (25)1:3-8.

26. SILVA VS, et al. Mobilização na Unidade de Terapia Intensiva: revisão sistemática. Fisioterapia e Pesquisa, 2014; 21(4):398-404.

27. SOUSA CDD, et al. Desfechos funcionais após craniectomia descompressiva secundária à acidente vascular encefálico. Fisioter Bras, 2020;21(1):39-48.

28. THOMAS S, MEHRHOLZ J. Health-related quality of life, participation, and physical and cognitive function of patients with intensive care unit-acquired muscle weakness 1 year after rehabilitation in Germany: the GymNAST cohort study. BMJ Open, 2018; 8(7).

29. ZANATA IL, et al. Avaliação fonoaudiológica para decanulação traqueal em pacientes acometidos por traumatismo cranioencefálico. CoDAS, 2016;28(6):710-716. 